\title{
Muon Acceleration: Introduction and Limiting Technologies
}

\author{
Alan D. Bross ${ }^{1}$ \\ Fermi National Accelerator Laboratory \\ P.O. Box 500 \\ Batavia, IL 60510 \\ USA \\ E-mail: brossefnal.gov
}

\begin{abstract}
An accelerator complex that can produce ultra-intense beams of muons presents many opportunities to explore new physics. These facilities are unique in that, in a relatively straightforward way, they can present a physics program that can be staged and thus move forward incrementally, addressing exciting new physics at each step. An intense cooled lowenergy muon source can be used to perform extraordinarily precise lepton-flavor violating experiments. These same muons can be accelerated and then stored in a race-track like storage ring to produce neutrinos for experiments to explore neutrino mixing with unprecedented precision, creating the so-called Neutrino Factory. Finally these muons could be accelerated to very-high energy to do energy-frontier physics with a muon collider. This paper will give an introduction to muon accelerator facilities and their physics capabilities and will then discuss some of the technologies that must be developed in order to make these "concepts" a reality.
\end{abstract}

10th International Workshop on Neutrino Factories, Super beams and Beta beams

Valencia, Spain

30 June-05 July, 2008

1 This work was supported by the Fermi National Accelerator Laboratory, which is operated by UniversitiesResearch Association, under contract No. DE-AC02-76CH03000 with the U.S. Department of Energy, 


\section{Introduction}

The international research program in accelerator and experimental physics using ultraintense muon beams offers the opportunity for a staged science program that can, at each step, yield extremely rich physics. An intense, cooled low-energy muon source can be used to perform extraordinarily precise lepton-flavor violating experiments. These same muons can be accelerated and then stored in a race-track like storage ring to produce neutrinos for experiments to explore neutrino mixing with unprecedented precision, creating the so-called Neutrino Factory. Finally these muons could be accelerated to very-high energy to do energy-frontier physics with a muon collider. Figure 1 shows a schematic of a Muon Accelerator Complex.

The Neutrino Factory and Muon Collider Collaboration (NFMCC) [1] with its international partners has been studying the design of Neutrino Factory and Muon Collider facilities now for well over a decade, publishing the first comprehensive study of a Muon Collider facility in 1996 [2]. The concept schematic that came out of this work is shown in Figure 1. Since the 1996 Snowmass report, there was a comprehensive update published in 2003 [3] on the collaboration's work on Neutrino Factories and Muon Colliders and recently the Muon Collider Task Force [4] at Fermilab has considered, in some detail, how a Muon Facility could evolve at Fermilab (Figure 2). Of the facilities that have been under study in the NuFact series of conferences, we see immediately that with respect to neutrino physics, the Neutrino Factory presents a unique opportunity. It yields the potential for the most precise measurements in the neutrino sector and can be part of a much broader physics program. Although an optimized $\beta$-Beam facility can offer the same reach in the neutrino sector as the Neutrino Factory, the $\beta$-Beam facility cannot expand its physics reach beyond the neutrino sector as can be done with a muon facility.

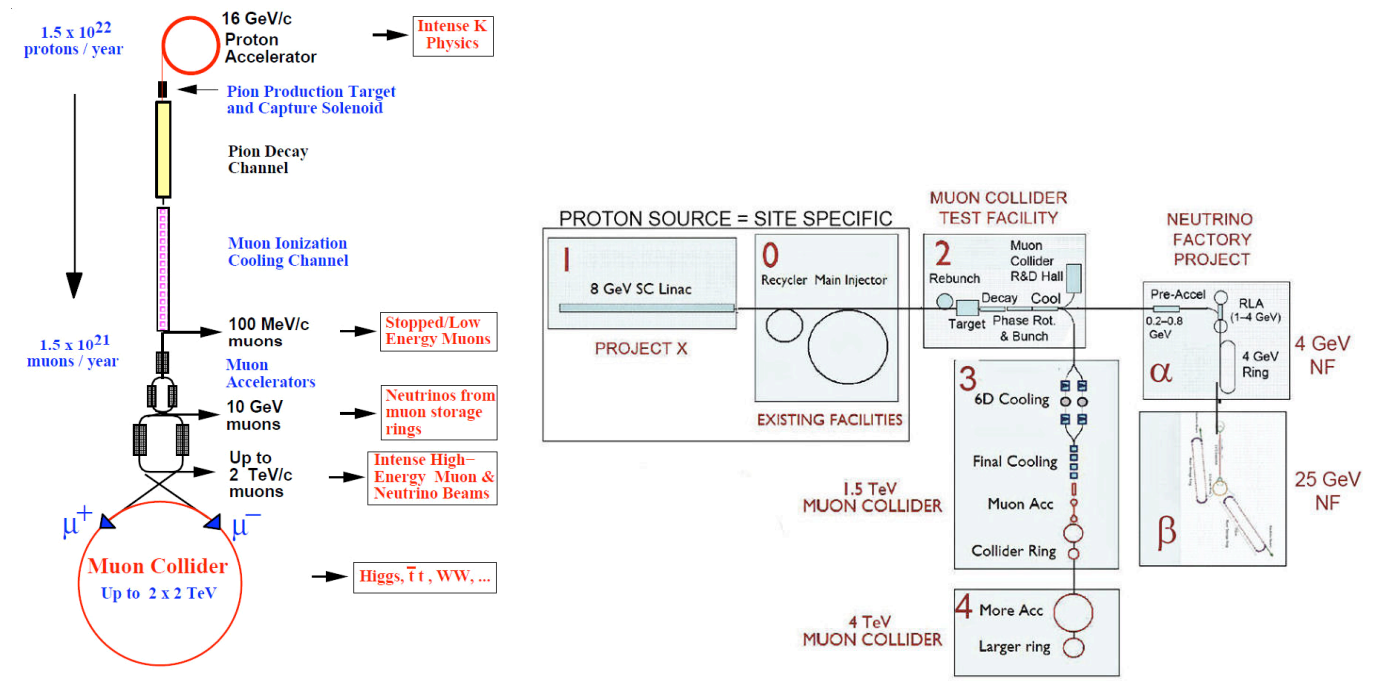

Figure 1 Schematic of Muon Accelerator Complex

Figure 2 Possible evolution of a Muon Facility 


\section{Neutrino Factory and Muon Collider Facilities}

As described in the introduction, current thinking indicates that the front end of the Neutrino Factory and the Muon Collider can be the same thus leading to interesting possibilities for staging. Another feature of these facilities is their small footprint, at least compared to energy-frontier $\mathrm{e}^{+} \mathrm{e}^{-}$colliders or hadron colliders. Figure 3 shows the baseline design for the Neutrino Factory from the International Scoping Study Report [5] and a concept for a $4 \mathrm{TeV}$ center-of-mass Muon Collider that can fit on the Fermilab site which is based on rapid-cycling synchrotron acceleration [6].

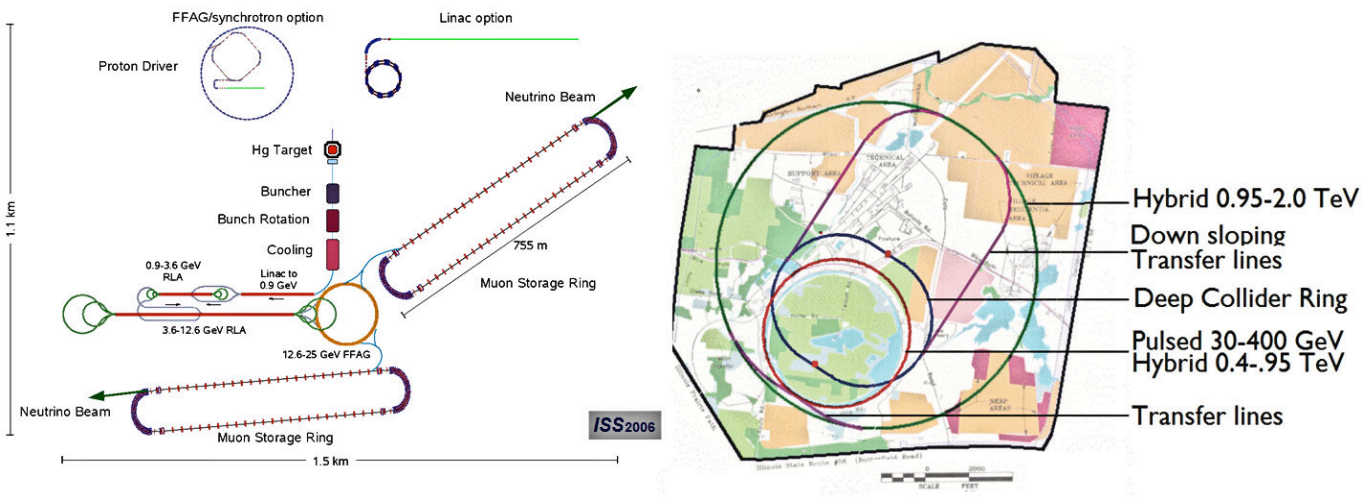

Figure 3 Neutrino Factory and Muon Collider Footprints

\subsection{Common Technological Needs}

Approximately $80 \%$ of the initial R\&D that is needed for the Neutrino Factory and the Muon Collider is common to both facilities. The following requirements are common:

1. Proton Driver The Fermilab option in this case is Project $X$ [7]. Reaching 4 MW beam power for a Neutrino Factory or a $1.5 \mathrm{TeV}$ center-of-mass Muon Collider is a significant challenge and beyond the Project $\mathrm{X}$ baseline.

2. Target, Capture and Decay A liquid $\mathrm{Hg}$ jet target is the baseline solution for both the Neutrino Factory and the Muon Collider.

3. Phase rotation In order to reduce the energy spread of the captured beam, phase rotation will be used.

4. Muon Ionization Cooling Transverse muon ionization cooling is needed in order to reduce the emittance of the muons. This is absolutely essential for the Muon Collider and is cost-effective for the Neutrino Factory.

5. Acceleration Acceleration based on low-frequency superconducting RF is utilized in both facilities in their baseline designs.

6. Storage Ring A ring capable of storing the muons for approximately 1000 turns is needed. Of course the storage ring for the Neutrino Factory is vastly simpler than the intersecting storage ring needed for the collider.

Although there is great synergy between the Neutrino Factory and the Muon Collider, there are key (and obvious) differences in the requirements for the two facilities. For 
the Muon Collider, much more cooling $\left(\mathrm{X} \mathrm{10}^{6}\right)$ is needed. Cooling in $6 \mathrm{D}$ is required, much more acceleration is used and, as mentioned above, the storage ring is an intersecting storage ring.

\subsection{Key R\&D Issues}

Many of the technologies and components for the Neutrino Factory and the Muon Collider are currently under study. The MERIT experiment [8] has successfully tested the concept of the liquid $\mathrm{Hg}$ jet target and it has shown very promising results which indicate that this type of target system can operate at a power level of $4 \mathrm{MW}$ and above. The Muon Ionization Cooling experiment [8] is preparing to perform a demonstration and engineering test of 4D cooling utilizing $200 \mathrm{MHz} \mathrm{RF}$ and liquid hydrogen absorbers. The MuCool [9] program is investigating operation of vacuum RF cavities in the presence of high magnetic fields, has made preliminary studies on liquid hydrogen absorbers and will also be studying the use of $\mathrm{LiH}$ absorbers in the early stages of muon cooling. The MuCool program focuses on component R\&D and, in addition to the capability to test RF components at high power, will have the capability to test cooling channel components with a high-intensity proton beam from the Fermilab linac. The Electron Model Muon Accelerator (EMMA) [10] experiment will study the properties of FFAGs which are a potential candidate for part of the acceleration system in both the Neutrino Factory and the Muon Collider.

Intense 6D cooling for the Muon Collider is not yet under experimental study, but is being modeled and studied with theoretical and computation tools. Concepts under study include the ring-FOFO or "Guggenheim", the Helical Cooling channel [11] and Parametric Resonance Ionization Cooling [12]. Figure 4 shows schematic concepts for the Guggenheim (left) and a helical solenoid (right) that is being developed for a proofof-principle test of components for the helical cooling channel.
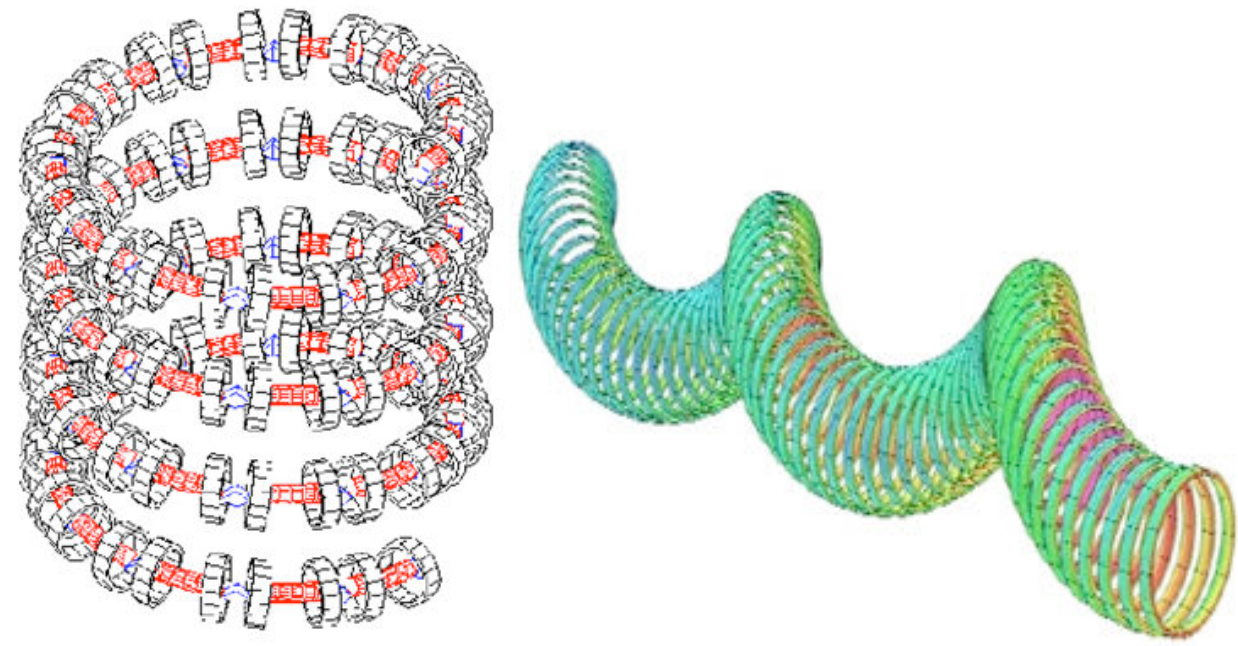

Figure 4 Guggenheim (left), Helical Solenoid (right) 


\subsection{RF Research and Development}

Of all the underlying accelerator technologies that are required for the Neutrino Factory and Muon Collider complexes, it can be argued that RF technology is the single most important "Limiting-Technology". It is of fundamental importance for these facilities in that it is needed in: 1. Muon capture, bunching and phase rotation, 2. Muon Cooling and 3. Acceleration. Both normal conducting RF (front-end, 1 and 2 above) and superconducting RF (acceleration) are required.

\subsubsection{Normal Conducting RF}

A crucial challenge for the Neutrino Factory and Muon Collider Front-End Design and cooling channels is the operation of high-gradient normal-conducting RF (NCRF) in the presence of high magnetic field. This problem has been the primary focus of the MuCool program. What has been observed in MuCool is that the safe operating gradient limit degrades significantly when a NCRF cavity is operated in magnetic field (Figure 5).

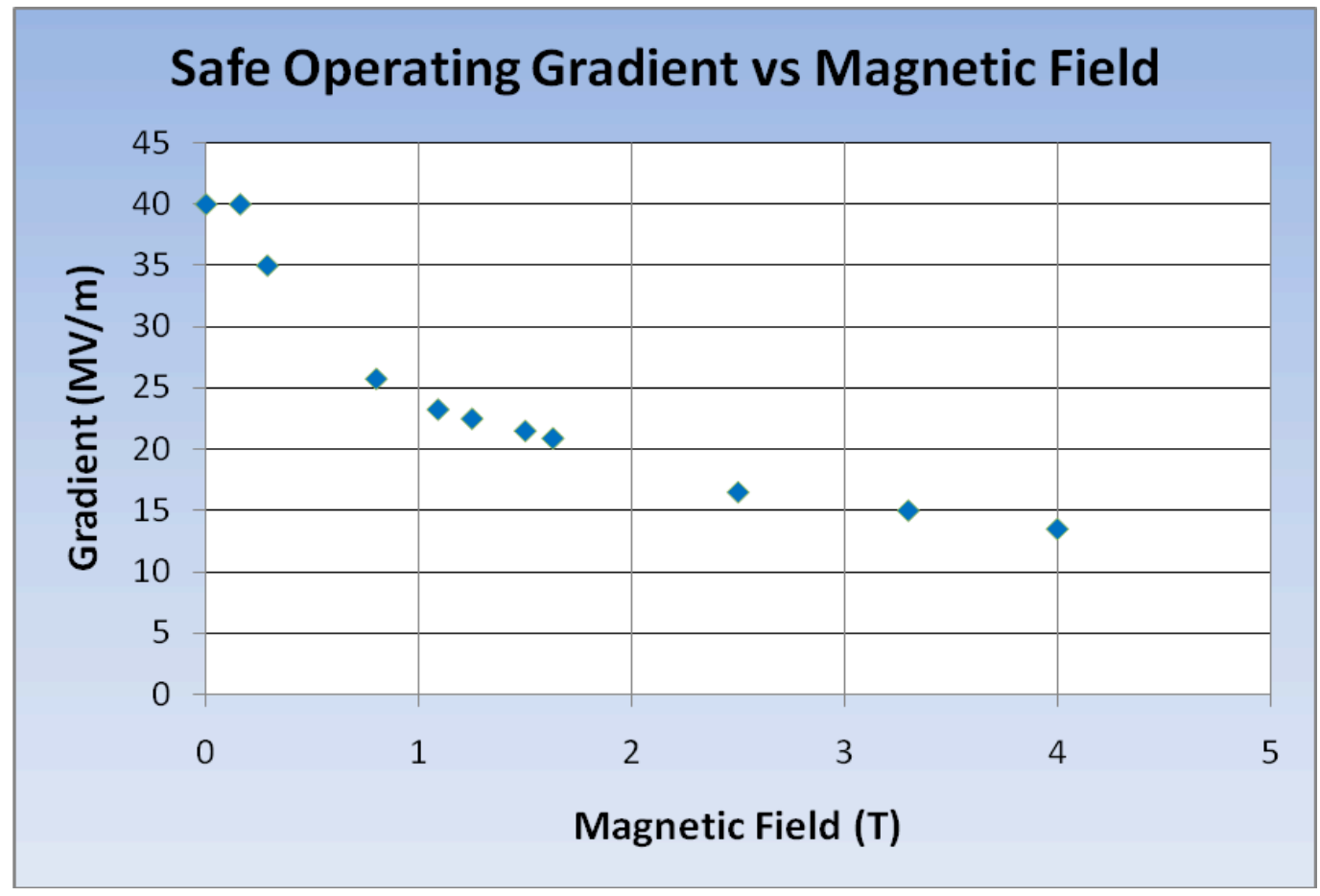

Figure 5 Maximum safe operating gradient vs. B field

The data shown in Figure 5 are for an $805 \mathrm{MHz}$ pillbox test cavity and seem to follow a universal curve. The maximum stable gradient degrades quickly with increasing $\mathrm{B}$ field. The cause of this effect is understood and comes from field emission from emitters (surface field enhancements in the regions of high gradient) in the cavity. The field emission is focused to spots by the B field when B is parallel to E as is the case with a pillbox cavity. This causes excessive heating and the surface to melt locally. Molten metal is accelerated away from the surface, vaporized and ionized by field 
emitted electrons, thus leading to breakdown of the cavity. In order to address this problem, three approaches are being investigated. The first is to eliminate field emission by processing the NCRF copper cavities using superconducting RF techniques. This has been done in MuCool for a $201 \mathrm{MHz}$ prototype copper cavity (prototype for MICE) with promising results. This cavity reached a stable operating gradient of $19 \mathrm{MV} / \mathrm{m}$ (the design gradient was $16 \mathrm{MV} / \mathrm{m}$ ) in the absence of B field. Initial tests of this cavity with applied external B field are encouraging, albeit the magnitude of the applied field to date has been low $(<<1 \mathrm{~T})$. A new concept in processing for SCRF which can, in principle, also be applied to NCRF is Atomic Layer Deposition (ALD) [13]. This is a CVD-like process that produces almost perfectly smooth surfaces. A single-cell $1.3 \mathrm{GHz}$ cavity (KEK shape) was prepared at Argonne National Laboratory and tested at Thomas Jefferson National Laboratory. The cavity test data equaled the best previous performance with this test cell, but in the test after the application of ALD, no field emission was detected [14] (Figure 6).
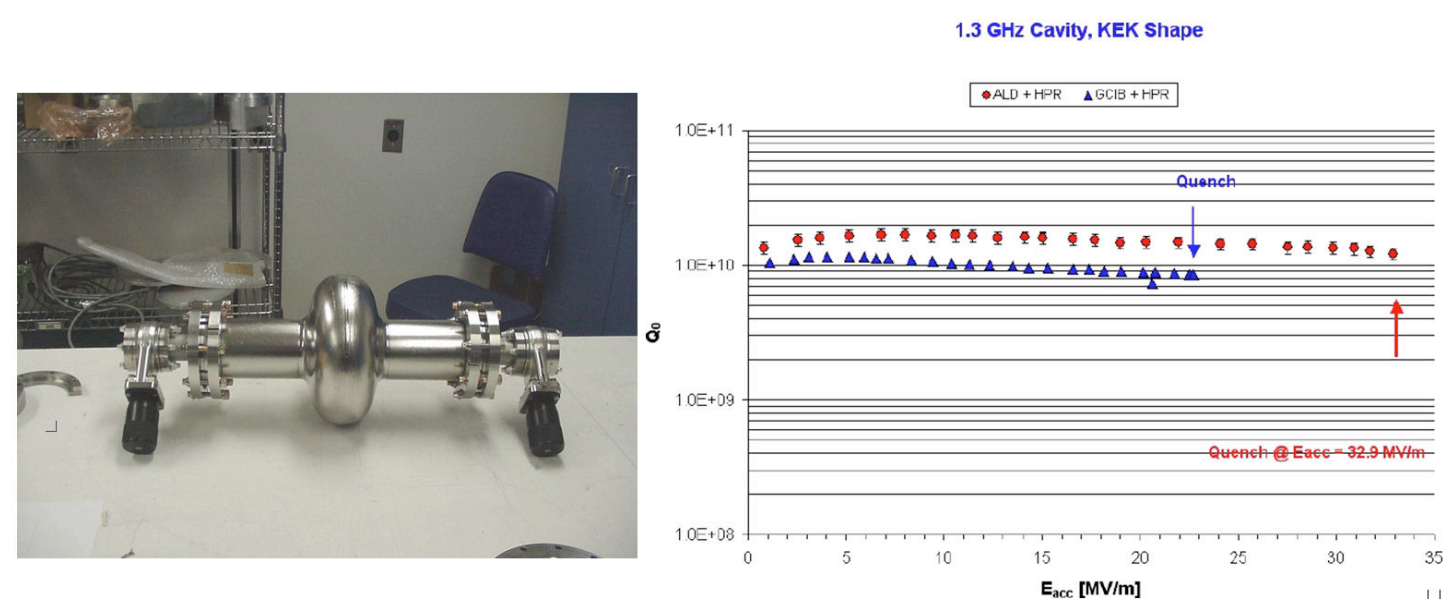

Figure $61.3 \mathrm{GHz}$ Test cell (left) Test results (right)

The second approach to abating the magnetic field effect is to prevent the magnetic focusing. One concept for this is shown in Figure 7. In this case an open cell geometry is required and additional coils are added (shown in purple in Figure 7) in the lattice to modify the B field direction and thus eliminate the B field focusing effect. However, the open-cell structure does mean that additional power (X2) is needed in order to reach the same on-axis accelerating gradient.

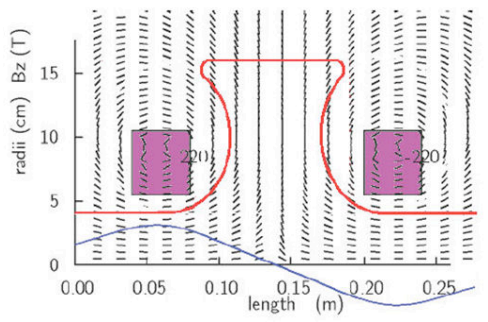

Figure 7 Magnetic Focusing

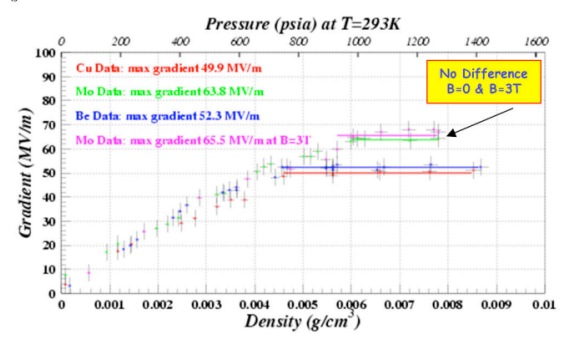

Figure 8 Data from $\mathrm{HP} \mathrm{H}_{2}$ filled RF Test Cell

The third approach to dealing with the magnetic field effect is to operate RF cavities filled with high pressure $\mathrm{H}_{2}$ gas. The results from tests in the MuCool Test Area done 
by our colleagues at Muons Inc [15] using a RF test cell filled with $\mathrm{H}_{2}$ gas are shown in Figure 8. In these data no degradation in maximum stable operating gradient was observed at B fields up to 3.5T for Mo electrodes.

\subsubsection{Superconducting RF}

Superconducting $201 \mathrm{MHz}$ RF is used in the early stages of acceleration in schemes for the Neutrino Factory and the Muon Collider that utilize re-circulating linear accelerators (RLA). Because of their large size (Figure 9) pure niobium cavities are not affordable, so explosion-bonded niobium on copper has been used for the cavity body [16]. Results from the first cavity of this type indicate that an accelerating gradient of 11 $\mathrm{MV} / \mathrm{m}$ can be achieved. However a large Q-slope (Figure 10) was observed in this cavity. The specification for these cavities is to reach $E_{a c c}>17 \mathrm{MV} / \mathrm{m}$, however. It is possible that the ALD process can be utilized for this type of cavity which, if the initial results on the solid niobium cavity is any indication (see above), may be able to address the Q-slope problem very effectively.

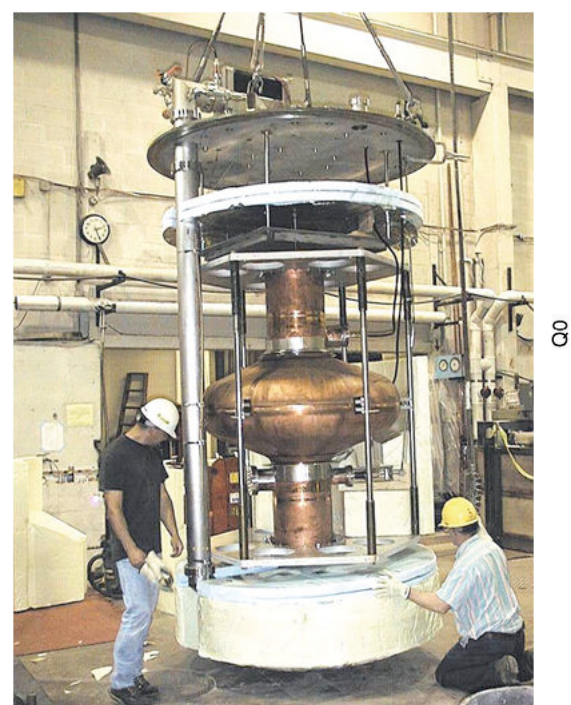

Figure $9201 \mathrm{MHz} S C R F$ cavity

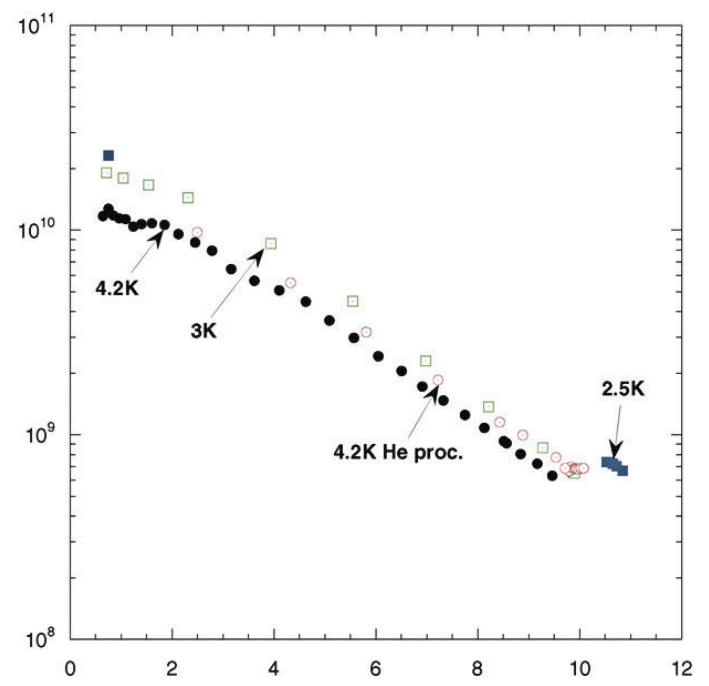

Figure $10 Q$ vs. Gradient for $201 \mathrm{MHz}$ SCRF cavity

\section{Conclusions}

There are many strong physics cases for facilities utilizing intense muon beams. Muon acceleration complexes are fundamentally different from other facilities addressing the neutrino sector or from other approaches to reach the energy frontier in that, in a natural way, a single facility can "evolve" such that it can address an extremely broad range of physics topics. The Neutrino Factory is a natural first step towards an energy-frontier Muon Collider which is a very exciting option to reach multi-TeV center-of-mass lepton-lepton collisions. In addition the ultra-intense muon source needed for the Neutrino Factory and the Muon collider could also be used for Lepton Flavor Violation experiments (muon-electron conversion, for example) of unprecedented sensitivity. 
Most technical issues for the Neutrino Factory are under study and have a good technical foundation at this time. Muon cooling is the crucial technical challenge for the Muon Collider, which stills needs a significant amount of work in order to put the Muon Collider on a similar technical foundation as the Neutrino Factory. The single most critical technical component issue for these facilities is high-gradient operation of RF cavities in large magnetic field.

\section{References}

[1] The MC collaboration website is at http://www.cap.bnl.gov/mumu/.

[2] Muon-Muon Collider: A Feasibility Study, BNL-52503, Fermilab Conf-96/092, LBNL-38946 (1996)

[3] Charles M. Ankenbrandt et al. (Muon Collider Collaboration), Phys. Rev. ST Accel. Beams 2, 081001 (1999)

[4] https://mctf.fnal.gov/

[5] RAL-TR-2007-019, RAL-TR-2007-23 and RAL-TR-2007-24

[6] Summers et al., “A Very Fast Ramping Muon Synchrotron for a Neutrino Factory”, in Proceedings of the 2003 Particle Accelerator Conference, pg 1804

[7] http://projectx.fnal.gov/

[8] H. Kirk, Experiments for accelerator $R \& D$, in proceedings of 10th International Workshop on Neutrino Factories, Super beams and Beta beams (NuFact08), June 30 -- July 5 2008, Valencia, Spain PoS(Nufact08)020.

[9] http://www.fnal.gov/projects/muon_collider/cool/cool.html

[10] C. Johnstone et al, "Hardware for a Proof-of-Principle Electron Model of a Muon FFAG", Nuclear Physics B - Proceedings Supplements, Volume 155, Issue 1, May 2006, Pages 325-327.

[11] T. Roberts et al., "MANX, A 6-D Muon Cooling Demonstration Experiment", in Proceedings of the Particle Accelerator Conference, 16-20 May, 2005, pg. 3331 - 3333.

[12] Y. Derbenev and R. Johnson, "Advances in Parametric-resonance Ionization Cooling,", in the Proceedings of 11th European Particle Accelerator Conference, Magazzini del Cotone, Genoa, Italy, 23-27 Jun 2008, pg. WEPP149.

[13] J. Norem et al., "Results from Atomic Layer Deposition and Tunneling Spectroscopy for Superconducting RF Cavities," in the Proceedings of 11th European Particle Accelerator Conference (EPAC 08), Magazzini del Cotone, Genoa, Italy, 23-27 Jun 2008, pp WEPP099.

[14] R. Rimmer, private communication.

[15] http://www.muonsinc.com

[16] R. Geng et al., "First RF Test at 4.2K of a 200MHz Superconducting Nb-Cu Cavity”, in Proceedings of the 2003 Particle Accelerator Conference, 2003, pg 1309. 